\title{
8 Wahrung der Patienten-Selbstbestimmung in der Notfallmedizin durch voraus- schauende Behandlungsplanung (Advance Care Planning)
}

Jürgen in der Schmitten, Stephan Rixen und Georg Marckmann

\subsection{Einleitung}

\section{Fallbeispiel 1}

Eine 83-jährige Frau, bisher Selbstversorgerin in eigener Wohnung, wird zur Abklärung von Durchfällen stationär eingewiesen. Dort zeigt sich in der Bildgebung ein vermutlich vom Eierstock ausgehender Tumor, der bereits an vielen Stellen in den Darm eingewachsen ist. Zur geweblichen Sicherung der Diagnose wird die Patientin operiert; die Diagnose wird intraoperativ bestätigt, die Operation ohne weiteren Eingriff beendet: Es handelt sich augenfällig um eine unheilbare Erkrankung, die nach ärztlichem Ermessen innerhalb weniger Wochen oder Monate zum Tode führen wird. Am Abend des Operationstages erleidet die Patientin auf der Intensivstation einen Herzstillstand. Sie wird reanimiert und überlebt. Eine Woche später erleidet sie einen Darmverschluss - sie wird erneut operiert, erhält einen künstlichen Darmausgang und überlebt. Eine Woche später wird sie erst auf die hauseigene Palliativstation, zwei Tage später ins nahegelegene Hospiz verlegt, wo sie tags darauf verstirbt.

\section{Fallbeispiel 2}

Ein 93-jähriger Mann lebt seit 15 Jahren im Altenheim. Durch mehrere Schlaganfälle ist er vollständig immobil und schwerst pflegebedürftig, zudem kann er nicht mehr sprechen. Seine Tochter besucht inn täglich und steht auch neben seinem Bett, als er einen Herzstillstand erleidet. Sie alarmiert das Pflegepersonal, dieses das Rettungsteam, und der Sterbende wird Gegenstand eines 45 Minuten dauernden erfolglosen Reanimationsversuchs. 


\section{Fallbeispiel 3}

Eine 78-jährige Frau lebt an der Seite ihres Mannes ein gesellschaftlich aktives, selbstbestimmtes Leben, bis sie einen schweren Schlaganfall erleidet, mit halbseitiger Lähmung sowie kompletter Schluck- und Sprachstörung. Der Notarzt wird gerufen, auf der Intensivstation (Stroke Unit) bestätigt sich ein ausgedehnter Hirninfarkt, eine Notfalltherapie (Lyse) ist nicht erfolgreich. In der Hoffnung auf eine zwar wenig wahrscheinliche, aber mögliche neurologische Verbesserung wird die Patientin zunächst durch Infusionen, später durch künstliche Ernährung über eine Magensonde am Leben erhalten. Doch die neurologische Frührehabilitation zeigt nicht die erhoffte Wirkung. Nach 6-wöchiger stationärer Therapie wird die nunmehr dauerhaft schwerstpflegebedürftige Patientin in ein Altenheim entlassen, wo sie bald wieder selbst schlucken kann und noch jahrelang bettlägerig lebt, ohne sich je wieder im herkömmlichen Sinne mitteilen oder fortbewegen zu können. Ihre Familie ist erschüttert und davon überzeugt: Das hätte unsere Ehefrau/Mutter/Schwester nie gewollt.

Diese Fallbeispiele stehen für unzählige andere - und niemand ermisst das Leid der betroffenen Patienten noch das der Angehörigen, die von solchen Erlebnissen oft für Jahre traumatisiert werden. Die ersten beiden Beispiele zeigen nur die - freilich besonders absurde - Spitze des Eisbergs, nämlich die Durchführung medizinisch unsinniger („nicht indizierter“) Behandlungen aufgrund von akutmedizinischen Handlungsroutinen, die nicht rechtzeitig hinterfragt wurden. Das dritte Beispiel deutet dagegen auf den noch um ein Vielfaches größeren (und komplexeren) Problemkreis, der sich öffnet, wenn man auch medizinisch Erfolg versprechende („indizierte“), aber von den Patienten angesichts gegebener Risiken nicht (mehr) gewollte Behandlungen betrachtet. Das Problem ist bekannt und wurde schon vor Jahren sehr treffend beschrieben (Salomon 2000).

Doch weder die in den letzten 20 Jahren erfolgte Etablierung der Palliativmedizin noch die seit über 40 Jahren propagierte und im Jahr 2009 gesetzlich gestärkte konventionelle Patientenverfügung scheinen dieser Fehlentwicklung etwas entgegensetzen zu können - namentlich nicht, trotz zunehmender Sensibilisierung für den Handlungsbedarf (Wiese et al. 2oogb), im Bereich der Notfallmedizin: „Herkömmliche Patientenverfügungen sind für die End-of-life-Care in der Intensivmedizin wenig hilfreich, denn sie werden in der Regel ohne ausreichende Kenntnis der intensivmedizinischen Wirklichkeit erstellt" (Hartog 2015). Im gleichen Beitrag wird darauf verwiesen, dass neben den unmittelbar Betroffenen und Angehörigen auch das betreuende ärztliche und pflegerische Personal schwer an dieser massiven Fehlentwicklung zu tragen hat. Aber darf es länger einfach hingenommen werden, dass alte, fragile und schwerkranke Menschen sowie zahlreiche mittelbar Betroffene in unserem Gesundheitssystem regelmäßig Opfer einer Fehlversorgung werden (von der Vergeudung solidarisch aufgebrachter Ressourcen nicht zu reden)? Und das, obwohl eine Lösung nach allem, was wir wissen, schon seit Jahren nur auf ihre - vermutlich sogar mindestens kostenneutrale (Klingler et al. 2015) - Umsetzung wartet?

Denn das im Folgenden skizzierte, in den vergangenen 20 Jahren entwickelte Konzept des Advance Care Planning (ACP) hält auch für die Notfallmedizin Mittel und Wege bereit, den Status quo ganz wesentlich zu verbessern. Voraussetzung für ein Verständnis dieses Potenzials ist die Bereitschaft, sich gedanklich von der in Deutschland seit Jahrzehnten zirkulär geführten Diskussion um die Patientenverfügung und ihren Problemen - gerade im Kontext der Notfallmedizin - zu lösen und sich auf etwas Neu- 
es, auf eine fundamental andere Herangehensweise einzulassen, die in Kapitel 8.2 kurz vorgestellt wird. Danach wird kurz auf die gerade in diesem Bereich auch von Experten oft falsch verstandene Rechtslage eingegangen (s. Kap. 8.3). Kapitel 8.4 präsentiert das Konzept des Notfallbogens im Kontext eines regionalen ACP-Programms am Beispiel der Hausärztlichen Anordnung für den Notfall (HAnNo) aus dem Projekt beizeiten begleiten. Speziell Interessierte finden an anderer Stelle weitere Informationen (in der Schmitten et al. 2011a und b; Rixen 2015).

\subsection{Vorausschauende Behandlungsplanung/Advance Care Planning: ein neues Konzept}

Vorausschauende Behandlungsplanung/Advance Care Planning folgt dem Anspruch, dass der Einsatz medizinischer Behandlung grundsätzlich am Wohl des Patienten zu orientieren ist (Patienten-Zentrierung). Ob eine Behandlung aber $\mathrm{zu}$ seinem Wohl ist oder nicht, bestimmt wo immer möglich am besten der Patient selbst (Patientenautonomie). Um zu einer solchen Festlegung in der Lage, also zu einer autonomen Entscheidung befähigt (in der Schmitten 2014) zu sein, muss er die Vor- und Nachteile der verschiedenen Behandlungsoptionen (einschließlich der Option, nichts zu tun) überblicken und bewerten können; dazu bedarf es eines intensiven Gesprächsprozesses (Prinzip des „Einverständnis nach Aufklärung“ oder informed consent). Folglich sollten Menschen Gelegenheit erhalten, mögliche künftige, mit Nicht-Einwilligungsfähigkeit einhergehende medizinische Krisensituationen vorab für sich durchzudenken und zu entscheiden, solange das noch möglich ist.

Vorausschauende Behandlungsplanung unterscheidet sich von der konventionellen Herangehensweise an Patientenverfügungen also grundlegend darin, dass sie den Gesprächsprozess zwischen dem Patienten, seinen Bevollmächtigten/Angehörigen (wo gewünscht und vorhanden) und einer dazu qualifizierten Gesundheitsfachperson in den Mittelpunkt stellt und somit dem bisher hier scheinbar außer Kraft gesetzten Prinzip des Informed Consent auch für den Bereich der Patientenverfügung prinzipiell Geltung verschafft. Die schriftliche Patientenverfügung wird zum Zwischenergebnis eines solchen Gesprächsprozesses, der im Laufe der Jahre - unter Berücksichtigung des Verlaufs etwaiger Erkrankungen des Individuums - regelmäßig oder bei Bedarf fortgeführt wird, ggf. mit entsprechender Anpassung der schriftlichen Verfügung. Der qualifizierte Gesprächsprozess führt somit zu verlässlichen (validen), aber auch zu für viele typische klinische (auch notfallmedizinische) Situationen aussagekräftigen Vorausentscheidungen. Eine geeignete, entsprechend differenzierte Dokumentation bildet diese individuellen Festlegungen angemessen ab.

Solcherart entstandene, verlässliche und aussagekräftige Patientenverfügungen auf differenzierten, regional einheitlichen Formularen müssen regelmäßig allen Menschen einer Zielgruppe ermöglicht und stets wo nötig aktualisiert werden, sie müssen bei Bedarf zur Hand sein und dann auch von den gerade Handelnden respektiert werden. Um dies zu gewährleisten und um die dafür erforderlichen personellen und finanziellen Ressourcen zu schaffen, setzt vorausschauende Behandlungsplanung die regionale Implementierung eines geeigneten strukturierten Systems voraus, das an einer definierten Zielgruppe orientiert ist, z.B. allen Altenheimbewohnern wie beim Projekt beizeiten begleiten (in der Schmitten u. Marckmann 2015) oder auch, wie in La Crosse/ 
USA beim Projekt Respecting Choices (Hammes u. Briggs 2015), allen Senioren ab dem 6o. Lebensjahr. Mit Blick auf die Ressourcen sei hier nur am Rande darauf hingewiesen, dass das sog. Hospiz- und Palliativgesetz, das im Herbst 2015 verabschiedet werden soll, im geplanten neuen $\mathbb{1} 132 \mathrm{~g}$ SCB V daran interessierten Pflegeeinrichtungen die Möglichkeit gibt, ein entsprechendes Angebot für ihre Bewohner mit Mitteln der Gesetzlichen Krankenversicherung zu finanzieren (Rixen 2015; Widmannn-Mauz 2015). Ein solches System der vorausschauenden Behandlungsplanung sollte folgende Elemente beinhalten, um funktionsfähig zu sein (ausführlich zu Advance Care Planning s. die Beiträge in Coors et al. 2015):

1. Aufsuchendes Gesprächsangebot

2. Qualifizierte Gesprächsbegleitung

3. Professionelle Dokumentation auf regional einheitlichen Formularen

4. Archivierung, Zugriff und Transfer von Vorausverfügungen

5. Aktualisierung/Fortführung des Gesprächsprozesses im Verlauf

6. Beachtung und Befolgung von Vorausverfügungen durch Dritte

7. Installierung eines Prozesses der kontinuierlichen Qualitätssicherung

\title{
8.3 Rechtsverbindlichkeit von Patientenverfügungen in der Rettungs- und Intensivmedizin
}

\begin{abstract}
Fallbeispiel 4
Nach einer Fortbildungsveranstaltung des Erstautors für Rettungsdienstmitarbeiter zum Thema Vorausschauende Behandlungsplanung erhebt sich ein altgedienter Notarzt und äußert unter beifälligem Gemurmel des Auditoriums: „Also, das ist ja alles schön und gut, was hier gesagt wurde, Verbindlichkeit von Patientenverfügungen und so. Aber ich kann den Teamkollegen nur empfehlen und werde selber weiter praktizieren: Wer zu einem $\mathrm{Pa}$ tienten mit Herzstillstand gerufen wird, der versucht zu reanimieren - egal, was in einer Patientenverfügung steht."
\end{abstract}

\section{Fallbeispiel 5}

Eine Verwandte des Erstautors erhält ein Hausnotrufsystem vom Deutschen Roten Kreuz (DRK). Sie hat eine Patientenverfügung und einen ärztlich unterschriebenen Notfallbogen, in dem ein Reanimationsversuch kategorisch abgelehnt wird. Darauf angesprochen äußert der verantwortliche DRK-Mitarbeiter (im Jahr 2015): „Das können Sie mir gerne zur Kenntnis geben, aber Sie sollten wissen, dass wir die Patientin immer reanimieren werden, wenn wir sie entsprechend vorfinden, ganz gleich, was sie verfügt hat. Dazu sind wir gesetzlich verpflichtet."

Die beiden Fallbeispiele stehen stellvertretend für ein historisch verständliches, heute aber problematisches Selbstverständnis vieler Mitarbeiter des Rettungsdienstes, das einer Lösung der eingangs geschilderten Missstände derzeit im Wege steht. Eine solche Haltung ist medizinethisch nicht akzeptabel, da sie rettungsdienst- anstatt patienten-zentriertes Denken offenbart, und sie ist mit der deutschen Gesetzeslage unvereinbar, sobald man nämlich das neue Konzept der vorausschauenden Behand- 
lungsplanung im Kontext der geltenden Rechtslage analysiert. Im Folgenden werden einige wichtige rechtliche Fragen geklärt, die seit der Verabschiedung des sog. Patientenverfügungsgesetzes (PVG) immer noch für Unsicherheit sorgen.

\subsection{1 (Not-)Ärztliche Befolgung von Patientenverfügungen in Ermangelung eines Vertreters}

Grundsätzlich sind die in einer Patientenverfügung (PV) vorausverfügten Behandlungswünsche eines Patienten auch in einer medizinischen Notfallsituation zu respektieren. Allerdings sind Rettungsdienst-Einsätze bei akut gefährdeten, nicht-einwilligungsfähigen Patienten meist dadurch gekennzeichnet, dass

- der vom Gesetz vorgesehene Vertreter nicht verfügbar ist (oder unter Schock steht),

- unabhängig davon die verfügbare Zeit eine Erörterung und gemeinsame Entscheidungsfindung ohnehin nicht zulässt und

- der vom Gesetz vorgesehene dialogische Abwägungsprozess zwischen Arzt und Vertreter zur Interpretation einer PV ( $\$ 1901$ b BCB) daher in der Regel situativ nicht oder allenfalls rudimentär möglich ist.

Für den Rettungsdienst ist daher von entscheidender Bedeutung, ob einer Patientenverfügung auch ohne aktive Beteiligung des Vertreters ärztlicherseits gefolgt werden darf, wenn darin - bezogen auf die konkret zu treffende Notfallentscheidung - eine eindeutige, nicht interpretationsbedürftige Festlegung erkennbar ist. Diese Frage ist im Gesetzestext nicht ausdrücklich beantwortet, in der anschließenden Fachdiskussion nach kurzer Irritation jedoch von maßgeblicher Seite unmissverständlich bejaht worden (vgl. Bundesärztekammer 2011; Wiese et al. 2009a sowie Kutzer 2010).

\subsubsection{Anwendbarkeit von Patientenverfügungen auf die Notfallsituation}

Selbst wenn Notärzte auch ohne Hinzuziehung eines Vertreters eindeutigen PVen formal folgen dürfen, verbleibt doch die praktische Schwierigkeit, innerhalb kürzester Zeit zu prüfen, ob die PV auf die vorliegende Situation zutrifft. Diese Schwierigkeit hat zwei Dimensionen:

- Zum einen ist es dem Rettungsdienst unter den Bedingungen einer Notfallsituation nicht zuzumuten, eine PV zu erfragen oder gar zu suchen sowie einen ausführlichen Verfügungstext auf der Suche nach einem situativ relevanten Passus zu studieren.

- Zum anderen lässt sich sagen, dass die in Deutschland bisher verbreiteten PVen in Notfallsituationen regelmäßig nicht anwendbar sind, da sie - wenn sie überhaupt konkret werden - meist Handlungsanweisungen an prognostische Einschätzungen knüpfen (wenn Zustand X mit Prognose Y, dann Handlung Z). In einer Notfallsituation sind jedoch weder die zur Prognoseeinschätzung nötigen Informationen noch die für die Interpretation eines solchen konditionierten Behandlungswunsches erforderliche Zeit und Ruhe verfügbar.

Um wirksam werden zu können, müssen PVen demzufolge (a) im Ernstfall vorliegen und (b) auf Anhieb klar ersichtliche, eindeutige Handlungsanweisungen beinhalten . 
Gut sichtbar aufbewahrte oder - in Institutionen - vom Pflegepersonal bereitgehaltene Notfallbögen (s.u. Kap. 8.4) können beide Bedingungen erfüllen und sind dann auch für den Rettungsdienst verbindlich.

\subsubsection{Validität: Kongruenz zwischen Patientenwille und -verfügung}

Um Missverständnisse zu vermeiden, sei vorab klargestellt: PVen sind rechtlich unabhängig davon gültig, d.h. bindend für notfallmedizinisches Handeln, ob ihre Validität zum Zeitpunkt der Anwendung für die Handelnden nachvollziehbar ist (zum Beispiel durch eine Unterschrift des beratenden Arztes) oder nicht. Aus medizinethischer und ärztlicher Perspektive muss jedoch hinzugefügt werden: Das Instrument der Patientenverfügung steht und fällt mit ihrer Validität, d.h. mit der Übereinstimmung dessen, was geschrieben steht (genauer: was ihre Adressaten aus ihr herauslesen), mit der tatsächlichen Verfügungsabsicht des Betreffenden für den entsprechenden Fall zum Zeitpunkt der Abfassung der PV. Dies setzt in der Regel eine qualifizierte Gesprächsbegleitung bei der Erstellung einer PV voraus.

Der Gesetzgeber hat davon abgesehen, eine solche qualifizierte und auf der PV dann durch ärztliche Unterschrift dokumentierte Beratung zur Pflicht zu machen: PVen erlangen vielmehr durch die einfache Unterschrift des Betroffenen Gültigkeit und müssen de jure - solange keine Hinweise auf einen Widerruf oder auf Manipulation vorliegen - befolgt werden, häufig ohne dass die Möglichkeit besteht, ihre Validität zu überprüfen. Gerade für das ärztliche und nicht-ärztliche Personal des Rettungsdienstes kann es eine extreme psychische Belastung und im Grunde unlösbare Aufgabe darstellen, mit einer PV konfrontiert zu sein, die bei vital bedrohlicher Krise ein Eingreifen trotz guter Prognose nicht erlaubt, ohne dass über die bloße (und überdies vielleicht schon lange zurückliegende) Unterschrift des Betroffenen hinausgehende Hinweise dafür vorliegen, ob dies tatsächlich ist, was der Patient gemeint hatte. Dringlich wünschenswert, wenn auch rechtsformal nicht geboten, ist es daher, Patientenverfügungen und speziell Notfallbögen so abzufassen, dass für den Anwender auf einen Blick eine stattgehabte qualifizierte Gesprächsbegleitung erkennbar wird.

\subsubsection{Notarzt, Notfallsanitäter (Rettungsassistent), Nachtwache ... an wen richten sich Patientenverfügungen?}

Der Adressat einer PV ist im PVG nicht ausreichend, jedenfalls nicht abschließend geregelt. Dort ist vielmehr nur vom „Arzt“ die Rede, dessen Stellung durch den Auftrag, die medizinische Indikation zu beurteilen und unter Berücksichtigung des verfügten oder mutmaßlichen Willens mit dem Vertreter zu diskutieren, zusätzlich bekräftigt wird. Dabei bleibt jedoch unberücksichtigt, dass es häufig nicht-ärztliches Personal ist, sowohl der Pflege (gerade in Institutionen wie Krankenhaus oder Altenheim) als auch des Rettungsdienstes, welches neben einer (Verdachts-)Diagnose auch die Indikation für die Einleitung mancher lebensrettender Behandlungsmaßnahmen zu stellen hat und ggf. vor dem Eintreffen des Arztes damit beginnen muss. Gerade die (Nicht-)Einwilligung in diese Maßnahmen, namentlich den Reanimationsversuch, ist jedoch fast regelmäßig Gegenstand von PVen. 
Nun ist die gesetzliche Regelung des PVG nicht abschließend zu verstehen, d.h., sie thematisiert nicht erschöpfend alle mit der Ausübung der Patientenselbstbestimmung berührten Fragen. Im Gegenteil: Bei der Gesetzesentstehung war unbestritten, dass die allgemeinen verfassungsrechtlichen Legitimationsgrundlagen und ihre strafrechtliche Absicherung weiterhin unverändert gelten (vgl. Bundestags-Drucksache 16/8442, S. 7-9). Das bedeutet, dass jeder Mensch auch außerhalb einer ArztPatienten-Beziehung von Verfassungs wegen (Art. 2 Abs. 1 und Abs. 2 S. 1 GG) befugt bleibt, darüber zu entscheiden, ob jemand, z.B. ein Notfallsanitäter (früher: Rettungsassistent) oder eine Pflegekraft, auf seinen Cesundheitszustand einwirken darf oder nicht. Die Bindung des Arztes an den Willen des Patienten, die im PVG, insbesondere in $\mathbb{\$} 1901 \mathrm{a}$ BGB, zum Ausdruck kommt, ist nur ein - wenn auch für das medizinische Geschehen sehr wichtiger - exemplarischer Fall autonomer Entscheidung des Einzelnen darüber, wer auf seinen Körper zugreifen und seinen Gesundheitszustand beeinflussen darf.

Aus dem Fehlen einer dies berücksichtigenden gesetzlichen Klarstellung darf nun nicht geschlossen werden, dass lebensrettende Maßnahmen von nicht-ärztlichem Personal unter Missachtung eindeutig anderslautender PVen, also wider besseres Wissen immer begonnen und bis zum Eintreffen des Arztes fortgesetzt werden müssten. Dass eine eindeutig zutreffende PV für den Arzt beachtlich ist, gilt daher der Sache nach - entgegen früheren anderslautenden Äußerungen aus der Rettungsmedizin (Wiese et al. 2011) - generell auch für nichtärztliches Personal, ohne dass dies im PVG geregelt wäre oder auch nur geregelt sein müsste (oder im Notfallsanitätergesetz- bzw. im früheren, inzwischen außer Kraft getretenen Rettungsassistentengesetz, auf das Wiese et al. [2011] sich berufen - wirksam abweichend geregelt sein könnte). Was verfassungsrechtlich u.a. im Verhältnis Patient/Notfallsanitäter (Rettungsassistent) garantiert ist, kann in Gesetzen, die unterhalb der Verfassung angesiedelt sind, nur klarstellend nachvollzogen werden, ohne dass es auf eine solche gesetzliche Klarstellung konstitutiv ankäme. Aus Gründen der Rechtssicherheit wäre es zwar zu begrüßen, wenn die Bindung von nicht-ärztlichem Personal an den vorausverfügten Patientenwillen durch eine gesetzliche Bestimmung explizit angeordnet würde. Das Notfallsanitätergesetz hebt zu Recht in einer nicht abschließenden Aufzählung eigenverantwortlich auszuführende Aufgaben des Notfallsanitäters hervor (vgl. $\$ 4$ Abs. 2 Nr. 1 Notfallsanitätergesetz), zu denen richtigerweise auch die Beachtung einer Patientenverfügung gehört. Allerdings ist eine solche wünschenswerte ausdrückliche gesetzliche Anordnung keine notwendige Voraussetzung für die allseitige rechtliche Bindungswirkung von Patientenverfügungen, weil diese Bindungswirkung bereits von Verfassungs wegen besteht.

Notfallsanitäter (Rettungsassistenten), die einer - soweit für sie erkennbar - korrekt ausgefüllten Hausärztlichen Anordnung für den Notfall (vgl. unten Kap. 8.4.3) folgend von einer Reanimation bei Herzstillstand absehen, handeln somit rechtmäßig. Würden sie sich hingegen über diese Verfügung hinwegsetzen und wider besseres Wissen dennoch eine Reanimation versuchen, müssten sie - nicht anders als Notärzte im gleichen Fall - damit rechnen, wegen Körperverletzung zur Rechenschaft gezogen zu werden. 


\subsection{Wirksame Vorausplanung in der Rettungs- und Intensivmedizin durch ärztliche Anordnungen für den Notfall (Notfallbögen)}

\subsubsection{Anforderungen an wirksame Vorausverfügungen in der Rettungs- und Intensivmedizin}

Angesichts der typischen Besonderheiten der Rettungs- und Intensivmedizin - Zeitnot, Stress, Entscheidungen auf Leben und Tod, Ausfall der Angehörigen, Entscheidungsnot auch für nicht-ärztliches Personal - stellt sich die Frage, was geschehen muss, damit PVen auch in diesem Bereich die von ihnen erhoffte Wirkung entfalten können. Die Antwort liegt auf der Hand: Patientenverfügungen müssen dafür

1. aussagekräftig sein, d.h. typische rettungs- und intensivmedizinische Entscheidungssituationen antizipieren und entscheiden,

2. erkennbar valide sein, d.h. für den Anwender nachvollziehbar größtmögliche Sicherheit bieten, dass in der Verfügung festgelegte Therapiegrenzen, deren Beachtung häufig in kürzester Zeit einen sonst unter Umständen vermeidbaren Todeseintritt zur Folge haben, auch tatsächlich dem Willen des Betroffenen entsprechen, sowie

3. klar und eindeutig formuliert sein, d.h. sich in der intendierten Bedeutung dem Anwender auch unter Zeitdruck auf Anhieb und unmissverständlich erschließen.

\subsection{2 Ärztliche Anordnungen für den Notfall}

Die Idee einer ärztlichen Anordnung für den Notfall wurde in den goer-Jahren in den USA entwickelt; dort sind die Physician Orders for Life-Sustaining Treatment (POLST) vielerorts bundesstaatliche amtliche Formulare (vgl. www.post.org) und haben in empirischen Untersuchungen ihre Eignung bewiesen (Hammes et al. 2012; Hickman et al. 2011). Auch in Deutschland gibt es schon länger Bestrebungen in diese Richtung (Kretschmer 2002; Wiese et al. 2008; Gerth et al. 2011), die konzeptionell jedoch meist ausdrücklich auf palliativmedizinische Situationen beschränkt sind und sich (daher?) bisher nicht durchgesetzt haben.

Für Entscheidungen über lebensrettende Behandlungsmöglichkeiten, die in kürzester Zeit getroffen werden müssen, ohne dass zuvor eine diagnostische Aufarbeitung der Situation erfolgen kann (d.h. meist: ohne dass eine individuelle Prognose möglich ist), erfüllt die Ärztliche Anordnung für den Notfall die im vorigen Abschnitt (s. Kap. 8.4.1) genannten Bedingungen - sofern die Verwendung dieses Bogens, diese unverhandelbare Bedingung wird leicht übersehen, in einen qualifiziert begleiteten Gesprächsprozess eingebettet ist:

- Die Aussagekraft der Notfall-Verfügung entsteht dadurch, dass der qualifizierte Gesprächsbegleiter den Betroffenen dabei unterstützt, notfall- und intensivmedizinische Szenarien sowie dabei potenziell zum Einsatz kommende Therapieoptionen zu verstehen und vor dem Hintergrund der individuell gegebenen Therapieziele zu entscheiden - und dass das Formular die Möglichkeit bietet, differenzierte Entscheidungen entsprechend abzubilden.

- Der gleiche Prozess gewährleistet auch die Validität, welche für den Anwender dadurch nachvollziehbar wird, dass neben dem Betroffenen auch der qua- 
lifizierte Gesprächsbegleiter und der den Gesprächsprozess begleitende Arzt die Formulare unterschreibt und damit attestiert, dass der Betroffene zu diesem Zeitpunkt einwilligungsfähig ist und die Implikationen seiner Festlegungen verstanden hat.

- Das Dokument eines regional einheitlichen, separat von der ausführlichen Vorausverfügung erstellten und als ärztliche Anordnung konzipierten Notfallbogens schließlich gibt auf einen Blick eine klare Auskunft darüber, ob in einer vital gefährdenden Krise mit dem Ziel der Lebensverlängerung behandelt werden soll und wenn ja mit welchen Mitteln.

Der Notfallbogen dokumentiert in weitestmöglicher Vereinfachung die gewünschten Behandlungsgrenzen für den Fall einer Krise, die sich aus dem aktuellen Zustand des Betroffenen heraus entwickelt. Der Notfallbogen gilt also nur für ein Szenario, nämlich das künftige Auftreten einer Krise im zum Zeitpunkt der Unterschrift gegebenen Ausgangszustand. Daraus folgt, dass nachhaltige chronische Veränderungen des Allgemeinzustands eine Überprüfung und ggf. Anpassung des Notfallbogens nach sich ziehen müssen.

Der Notfallbogen gibt dem Arzt zunächst die Möglichkeit, in Absprache mit dem Patienten oder seinem Vertreter Behandlungsoptionen auszuschließen, die medizinisch nicht mehr indiziert (weil für das gegebene Behandlungsziel nicht mehr erfolgversprechend) sind. Bei chronisch multi-morbiden, immobilen und schwerst pflegebedürftigen Menschen gilt dies zum Beispiel für einen Reanimationsversuch im Fall eines Herzstillstands (Hayes 2013).

Darüber hinaus berücksichtigt der Arzt in seiner Anordnung die vom Patienten (bzw. vom Vertreter im Namen des Patienten) festgelegten, individuell gewünschten Behandlungsgrenzen, die im Notfall gelten sollen. Die Erfahrung zeigt, dass die allermeisten Menschen im berufstätigen Alter im Notfall keine Einschränkung einer medizinisch indizierten, lebensrettenden Notfalltherapie wünschen; bei diesen mobilen Menschen hätte der Notfallbogen meist auch praktisch geringe Bedeutung, da er im Bedarfsfall nicht ohne weiteres zur Hand wäre. Im Renten- und höheren Alter zeigt die Erfahrung dagegen, dass die Einschränkung der Teilhabemöglichkeit mit den Grenzen lebensverlängernder Therapie korreliert, jedoch keineswegs ausnahmslos: Nicht wenige rüstige, mobile ältere Menschen schließen einen Reanimationsversuch bei Herzstillstand (sowie manchmal sogar weniger schwerwiegende Interventionen) aus, und umgekehrt gibt es hochbetagte, funktionell erheblich eingeschränkte Menschen, welche wünschen, dass ungeachtet schwindender Erfolgsaussichten alle Möglichkeiten der modernen Akutmedizin ausgeschöpft werden. Vorausschauende Behandlungsplanung ist mit einer sog. Reichweitenbeschränkung von PVen nicht vereinbar (vgl. Rixen 2015; in der Schmitten u. Marckmann 2013); der Notfallbogen ist ein wichtiges Instrument, um medizinisch indizierte, aber individuell nicht mehr gewünschte Behandlungsmaßnahmen wirksam abzulehnen.

Der Notfallbogen wird durch die Unterschrift des Betroffenen (bzw. Angehörigen) zu einer rechtsverbindlichen Patientenverfügung (bzw. Festlegung des Vertreters) im Sinne des PVG. Die zusätzliche Unterschrift des behandelnden Arztes attestiert für die in der Krisensituation handelnde Fachperson Einwilligungsfähigkeit und Verständnis der Implikationen seitens des Patienten, somit die Validität der Verfügung. 
Um Missverständnisse und Fehlinterpretationen durch die Anwender zu vermeiden, die sich trotz gemeinsamer Kriterien aus unterschiedlichen Formularen (mit unterschiedlichen Reihenfolgen, Optionen, sprachlichen und graphischen Konstruktionen etc.) ergeben können, empfehlen sich dringend regional einheitliche Formulare für PVen und insbesondere für Ärztliche Anordnungen für den Notfall.

\subsubsection{Die Hausärztliche Anordnung für den Notfall ( $\left.\mathrm{HAnNo}^{\circledR}\right)$ im Projekt beizeiten begleiten ${ }^{\circledR}$}

Ein solcher für die einheitliche regionale Verwendung konzipierter Notfallbogen ist die Hausärztliche Anordnung für den Notfall (HAnNo, s. Abb. 6), die für das Modellprojekt beizeiten begleiten (in der Schmitten u. Marckmann 2015; vgl. auch www.beizeitenbegleiten.de) entwickelt worden ist. Sie entsteht grundsätzlich am Ende eines mehrzeitigen und im Mittel mindestens einstündigen (sic!) Gesprächsprozesses, der durch einen beizeiten begleiten-zertifizierten Gesprächsbegleiter moderiert wurde und an dessen Abschluss in der Regel der Hausarzt prüfend und ggf. ergänzend oder revidierend beteiligt war; beide unterschreiben die HAnNo neben dem Patienten und/ oder dessen Vertreter. Das Ankreuzen einer Behandlungsoption auf der HAnNo repräsentiert also eine im Voraus für einen hypothetischen Fall ausgesprochene (Nicht-) Einwilligung, die dem Standard des Informed Consent so nahe kommt wie möglich. Anwender in Pflege, Rettungsdienst und Krankenhaus einer Region, in der beizeiten begleiten etabliert ist, können sich auf diesen zertifizierten Gesprächs-Qualitätsstandard der Entstehung einer HAnNo (und der dazugehörigen PV) verlassen. Darüber hinaus ist dieses Personal im Rahmen der Implementierung von beizeiten begleiten darin geschult worden, das Formular HAnNo einheitlich zu verstehen, adäquat zu archivieren, bei Patienten-Transfers mitzugeben und, last but not least, zu befolgen .

Daher wird (vom Schutz der Wort-Bild-Marke einmal abgesehen) an dieser Stelle auch ausdrücklich davor gewarnt, Nachahmungen des abgebildeten Muster-Formulars ohne eine solche systematische regionale Implementierung eines Advance Care Planning Programms zu verwenden, da andernfalls mit Missverständnissen und Fehlern gerechnet werden muss, die unabsehbare, deletäre Folgen haben können.

Die HAnNo kommt (wie jede PV) nur zur Anwendung, wenn der Betroffene aktuell nicht selbst entscheiden kann, und sie regelt explizit nur das notfallmäßige Vorgehen im Fall einer lebensbedrohlichen Krise. Für weniger zeitkritische Entscheidungen sind die ausführliche PV und ggf. der Vertreter (Bevollmächtigter bzw. Betreuer) heranzuziehen. Aus diesem Grund wird in der HAnNo - anders als in den POLST-Formularen mehrerer US-Staaten - u.a. weder die Frage einer PEG-Sonde noch die der Gabe von Antibiotika behandelt.

Der Optionsteil der HAnNo zeichnet sich in formaler Hinsicht dadurch aus, dass (anders als in den gängigen US-POLST-Formularen) nur genau ein (1) Kreuz gesetzt werden kann; dadurch werden auf POLST- und manchen deutschen Notfallbögen mögliche, aber klinisch unsinnige Kombinationen (z.B. Reanimation ja, aber Krankenhauseinweisung nein) ausgeschlossen. Inhaltlich gibt es drei Kategorien (A, B und C), die am Therapieziel und, im Fall von (B), darüber hinaus an den Belastungen und Risiken von klinisch relevanten Behandlungsoptionen orientiert sind; das farbliche Ampelschema (grün-rot, auf der schwarz-weißen Abbildung nicht erkennbar) erleichtert visuell das Verständnis: 
8.4 Wirksame Vorausplanung in der Rettungs- und Intensivmedizin durch ärztliche Anordnungen für den Notfall (Notfallbögen)

\section{Hausärztliche Anordnung für den Notfall HAnNo}

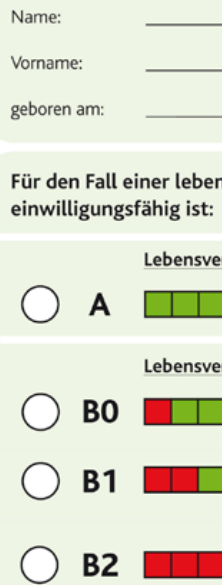

B1

B2

A \begin{tabular}{l|l|l|l|l}
\hline & & & & \\
Notfall- und Intensivtherapie einschließlich Herz-Lungen-Wiederbelebung
\end{tabular} Lebensverlängernde Therapie, aber mit folgenden Einschränkungen ( $\mathrm{BO}$ bis $\mathrm{B} 3$ ):

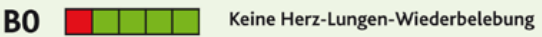

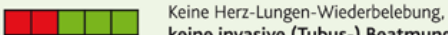
keine invasive (Tubus-) Beatmung Keine Herz-Lungen-Wiederbelebung, keine invasive (Tubus-) Beatmung, keine Behandlung auf Intensivstation keine invasive (Tubus-) Beatmung, keine Behandlung auf Intensivstation, keine Mitnahme ins Krankenhaus (aber ambulante Therapie) Keine Therapie mit dem Ziel der Lebensverlängerung, auch nicht ambulant (C): ○ C \begin{tabular}{|l|l|l|l|l}
\hline & & & & \\
Ausschließlich palliative (lindernde) Maßnahmen
\end{tabular}

\begin{tabular}{|c|c|}
\hline \multirow{4}{*}{$\begin{array}{l}\text { den } \_ \text {Ich bestätige Einwilligungsfähigkeit } \\
\text { und Verständnis der Implikationen dieser } \\
\text { Entscheidung." }\end{array}$} & "Diese HAnNo ist Ausdruck meines Behandlungswillens." \\
\hline & den $\ldots \cdot{ }_{-} \cdot 20$ \\
\hline & $\begin{array}{l}\boldsymbol{X} \\
\text { Unterschrift des Betroffenen (bei Einwilligungsfähigkeit) }\end{array}$ \\
\hline & $\begin{array}{l}\text { "Diese HAnNo gibt den (mutmaßlichen) Behandlungswillen } \\
\text { des Betroffenen angemessen wieder." }\end{array}$ \\
\hline $\mathbf{X}$ & $\begin{array}{l}\boldsymbol{X} \\
\text { Unterschrift und NAME des Vertreters/Angehörigen }\end{array}$ \\
\hline $\begin{array}{l}\text { Unterschrift und Stempel } \\
\text { des zertifizierten Hausarztes }\end{array}$ & $\begin{array}{l}\text { "Ich habe den Entscheidungsprozess begleitet." } \\
\boldsymbol{X} \\
\text { Unterschrift und NAME des zertifizierten Begleiters }\end{array}$ \\
\hline
\end{tabular}

Diese HAnNo ist der notfallmedizinisch relevante Teil der Patientenverfügung / Vertreterverfügung (Nichtzutreffendes streichen) vom __. . . $20 \ldots$ der ein qualifizierter Beratungsprozess gemäß beizeiten begleiten-Standard zugrundeliegt. Die vorliegende HAnNo reflektiert den (mutmaßlichen) Willen des Bewohners und ist - wenn vollständig und eindeutig ausgefüllt - für jedermann ethisch und rechtlich verbindlich, sofern der Bewohner nicht selbst einwilligungsfähig ist. Zur Klärung anderer Behandlungsfragen ist die ausführliche Verfügung heranzuziehen und ggf. der Vertreter (Bevollmächtigte bzw. Betreuer) zu konsultieren. Weitere Hinweise zur HAnNo siehe Rückseite.

- Option A entspricht dem uneingeschränkten Einsatz aller medizinisch vertretbaren Mittel zur Erreichung des Therapieziels der Lebensverlängerung und damit dem gängigen akutmedizinischen Standard, der grundsätzlich auch dann zur Anwendung kommt, wenn keine Vorausverfügung vorliegt. 
- Option B bejaht ebenfalls die Orientierung am Therapieziel der Lebensverlängerung, jedoch „nicht um jeden Preis“; vielmehr besteht die Möglichkeit, vier klinisch besonders relevante Behandlungen kumulativ auszuschließen, die durch spezielle, im Voraus gut zu würdigende Risiken bzw. qualitative Prognosesprünge gekennzeichnet sind:

- die kardiopulmonale Reanimation (Bo),

- zusätzlich die Tubusbeatmung (mit einer im Unterschied zur von diesem Ausschluss nicht berührten nicht-invasiven Beatmung deutlich schlechteren Prognose-B1),

- zusätzlich die Behandlung auf Intensivstation (B2) sowie

- zusätzlich jegliche stationäre Einweisung mit dem Ziel der Lebensverlängerung (B3).

- Option C unterscheidet sich grundsätzlich von den beiden vorgenannten Optionen, indem das Therapieziel der Lebensverlängerung explizit verneint wird. Die Auswahl dieser Option kann Ausdruck davon sein, dass der Versuch einer Lebensverlängerung in diesem Fall medizinisch unsinnig wäre, oder auch davon, dass Lebensverlängerung zwar medizinisch als aussichtsreich gelten dürfte, aber vom Patienten nicht mehr gewünscht wird.

Die regionale Implementierung eines ACP-Programms wie beizeiten begleiten bedeutet einen tiefgreifenden Eingriff in die medizinische Versorgungskultur („cultural change“), der über kurz oder lang unweigerlich Anregungen und Veränderungen in benachbarten Bereichen nach sich ziehen wird. Hier ist in erster Linie an Strukturen der ambulanten palliativmedizinischen Versorgung zu denken, denn die bisherige Praxis der stationären Einweisung mancher schwerkranker Menschen kann auch unter dem Aspekt eines strukturellen Defizits in diesem Bereich betrachtet werden. Zu den hierdurch aufgeworfenen Fragen zählen neben der Erreichbarkeit von Hausärzten außerhalb der Sprechstunde und der Qualifikation sowie fallbezogenen Informiertheit von Bereitschaftsärzten nicht zuletzt ein palliativmedizinisches Konzept für den Rettungsdienst, wie es bereits Gegenstand aktueller Forschung ist (Wiese et al. 2011), sowie dessen Würdigung im Rahmen der bisher meist einseitig auf das Therapieziel der Lebensrettung fokussierten Rettungsdienstgesetze der Länder.

Abschließend ist darauf hinzuweisen, dass auch die HAnNo nicht jeden (konstruierten) Anwendungsfall vollständig abdeckt - das ist auch nicht der Anspruch. Zudem lehrt die Erfahrung, dass selbst dieser vermeintlich eindeutig gestaltete Bogen nicht „selbsterklärend“ ist, sondern regional eingeführt und allen Beteiligten vorab bekannt gemacht werden muss, wenn ein reibungsloser Ablauf gewährleistet werden soll. Ein Notfallbogen kann folglich nur so gut bzw. erfolgreich sein wie die regionale Implementierung des Advance Care Planning Programms, dessen Teil er ist, und das bedeutet: wie die Schulung und Mitwirkung aller beteiligten Akteure, insbesondere der Gesprächsbegleiter und in der Folge der von ihnen moderierte Gesprächsprozess. Auch lässt das Formular, das naturgemäß einen Mittelweg zwischen Prägnanz und Vollständigkeit zu suchen hat, manche Lücken, für deren Diskussion hier kein Raum gegeben ist; exemplarisch sei an dieser Stelle das hoch sensible, in der Literatur zu Patientenverfügungen praktisch noch nicht berührte Thema einer iatrogenen Komplikation genannt, deren notfallmäßige Behebung mit der Festlegung in der HAnNo (oder allgemeiner: in der PV) zu konfligieren scheint. Auch liegt auf der Hand, dass für die einvernehmliche Durchführung elektiver Eingriffe wie Herz- 
katheter oder Cholezystektomie gesonderte Absprachen für die Notfallbehandlung in der peri-interventionellen bzw. -operativen Phase getroffen werden müssen. Grundsätzlich gilt: Patienten haben ein Recht darauf, die Grenzen ihrer Behandlungen selbst zu bestimmen - auch für den Fall künftiger gesundheitlicher Krisen. Extremfälle oder spezielle Situationen können Anreiz sein, bestehende Regelungen zu verbessern und Lücken zu schließen, dürfen jedoch nicht dazu missbraucht werden, praktikable Regelungen zu verhindern, mit denen die Beachtung des Patientenwillens im Regelfall sehr viel besser als bisher ermöglicht werden können.

\subsection{Fazit}

Patientenverfügungen sind auch in der Rettungs- und Intensivmedizin für alle Beteiligten verbindlich und jedenfalls dann relevant, wenn sie eine konkrete, auf die Situation zutreffende Behandlungspräferenz formulieren. Dringlich wünschenswert ist, dass solchen Verfügungen regelmäßig ein qualifizierter Gesprächsprozess zugrundeliegt und durch ärztliche Unterschrift für den Anwendbar nachvollziehbar dokumentiert ist. Internationale Erfahrungen und erste Ansätze in Deutschland zeigen, dass regionale Projekte vorausschauender Behandlungsplanung realisierbar sind und unter anderem ärztlich mitverantwortete Notfallbögen ermöglichen, welche die für Krisensituationen gebotenen Anforderungen an Eindeutigkeit, Aussagekraft und Verlässlichkeit erfüllen. Es liegt in der Hand der regionalen Akteure des Gesundheitswesens, durch Einführung solcher regionaler Projekte Voraussetzungen für eine wirksame vorausschauende Behandlungsplanung zu schaffen, die sich auch im Krisenfall bewährt - und dazu führt, dass auch im Bereich der Notfallmedizin Patientenzentrierung zur Regel und Fallbeispiele wie eingangs geschildert zur Ausnahme werden.

Dieser Beitrag basiert auf folgender Publikation derselben Autoren (Abdruck mit freundlicher Genehmigung des Kohlhammer Verlags):

Vorausplanung in der Notfall- und Intensivmedizin. In: Coors M, Jox RJ, in der Schmitten I (Hrsg.): Advance Care Planning. Von der Patientenverfügung zur gesundheitlichen Vorausplanung. Kohlhammer Verlag 2015, S. 288-301.

\section{Literatur}

Bundesärztekammer (2011) Grundsätze der Bundesärztekammer zur ärztlichen Sterbebegleitung. Deutsches Ärzteblatt 108(7): A-346/B-278/C-278

Coors M, Jox R, in der Schmitten I (Hrsg.) (2015) Advance Care Planning: Von der Patientenverfügung zur gesundheitlichen Vorausplanung. Kohlhammer: Stuttgart

Gerth MA, Mohr M, Paul N, Werner C (2011) Präklinische Notfall-Patientenverfügung - eine Evaluation aus der Sicht des Hausarztes. Zeitschrift für Allgemeinmedizin 87: 5

Hammes B, Briggs L (2015) Das „Respecting Choices“ Advance-Care-Planning-Programm in den USA: ein nachgewiesener Erfolg. In: Coors M, Jox R, in der Schmitten I (Hrsg.) Advance Care Planning: Von der Patientenverfügung zur gesundheitlichen Vorausplanung. Kohlhammer: Stuttgart, S. 181-195

Hammes BI, Rooney BL, Gundrum JD, Hickman SE, Hager N (2012) The POLST program: a retrospective review of the demographics of use and outcomes in one community where advance directives are prevalent. I Palliat Med 15(1): 77-85 
Hartog CS (2015) Grenzen der Vorausverfügung in der Intensivmedizin. In: Coors M, Jox R, in der Schmitten J (Hrsg.) Advance Care Planning: Von der Patientenverfügung zur gesundheitlichen Vorausplanung. Kohlhammer: Stuttgart, S. 52-61

Hayes B (2013) Clinical model for ethical cardiopulmonary resuscitation decision-making. Internal Medicine Journal 43(1): 77-83

Hickman SE, Nelson CA, Moss AH, Tolle SW, Perrin NA, Hammes BI (2011) The consistency between treatments provided to nursing facility residents and orders on the physician orders for life-sustaining treatment form. I Am Geriatr Soc 59(11): 2091-9

in der Schmitten I (2014) Autonomie gewähren genügt nicht - Patienten-Selbstbestimmung bedarf aktiver Förderung durch Ärzte. Zeitschrift für Allgemeinmedizin 90(6): 246-250

in der Schmitten I, Marckmann G (2013) Sackgasse Patientenverfügung. Neue Wege mit Advance Care Planning am Beispiel beizeiten begleiten ${ }^{\circledR}$. [Traditional advance directives - a dead end. Walking new ways with the advance care planning program beizeiten begleiten ${ }^{\circledR}$ ]. Zeitschrift für Medizinische Ethik 59: 229-243

in der Schmitten J, Marckmann G (2015) Das Pilotmodell beizeiten begleiten. In: Coors M, Jox R, in der Schmitten I (Hrsg.) Advance Care Planning: Von der Patientenverfügung zur gesundheitlichen Vorausplanung. Kohlhammer: Stuttgart, S. 234-257

in der Schmitten J, Rixen S, Marckmann G (2011a) Patientenverfügungen im Rettungsdienst (Teil 1). Geklärte und offene Fragen nach Verabschiedung des Patientenverfügungsgesetzes. Notfall Rettungsmed 14(6): 448-458

in der Schmitten I, Rothärmel S, Rixen S, Mortsiefer A, Marckmann G (2011b) Patientenverfügung im Rettungsdienst (Teil 2). Neue Perspektiven durch Advance Care Planning und die Hausärztliche Anordnung für den Notfall. Notfall Rettungsmed 14(6): 465-474

Klingler C, in der Schmitten I, Marckmann G (2015) Does facilitated Advance Care Planning reduce the costs of care near the end of life? Systematic review and ethical considerations. Palliat Med, 2015. I-II. (in print)

Kretschmer B (2002) Der „rote Umschlag“ für den Notarzt. Möglichkeiten und Chancen eines Notfallbogens als komprimierter Patientenverfügung im Rettungseinsatz. In: May AT, Geißendörfer SE, Simon A, Strätling M (Hrsg.) Passive Sterbehilfe: Besteht gesetzlicher Regelungsbedarf? Lit Verlag: Münster, S. 141-172

Kutzer K (2010) Ärztliche Pflicht zur Lebenserhaltung unter besonderer Berücksichtigung des neuen Patientenverfügungsgesetzes. MedR 28: 531-533

Rixen S (2015) Rechtliche Aspekte der vorausschauenden Behandlungsplanung (Advance Care Planning). In: Coors M, Jox R, in der Schmitten I (Hrsg.) Advance Care Planning: Von der Patientenverfügung zur gesundheitlichen Vorausplanung. Kohlhammer: Stuttgart, S. 164-180

Salomon F (2000) Entscheidungskonflikte am Notfallort. Anästhesiol Intensivmed Notfallmed Schmerzther 35(5): 319-325

Widmannn-Mauz A (2015) Geleitwort. In: Coors M, Jox R, in der Schmitten I (Hrsg.) Advance Care Planning: Von der Patientenverfügung zur gesundheitlichen Vorausplanung. Kohlhammer: Stuttgart, S. 9-10

Wiese CHR, Bartels U, Geyer A, Duttge G, Graf BM, Hanekop GG (2008) Göttinger Palliativkrisenbogen: Verbesserung der notfallmedizinischen Versorgung von ambulanten Palliativpatienten. „Die Gelbe Karte für den Rettungsdienst“. Dtsch Med Wochenschr 33(18): 972-976

Wiese CHR, Duttge G, Weber AK, Zausig YA, Ruppert D, Hanekop GG, Graf BM (2009a) Notfallmedizinische Betreuung von Palliativpatienten am Lebensende. Juristische Beurteilung notfallmedizinischer Handlungsweisen - retrospektive Fallbetrachtung zur medizinischen Indikation und zum Patientenwillen. Anaesthesist 58: 1097-1106

Wiese CHR, Ittner KP, Graf BM, Lassen CK (2011) Palliative Notfälle - Definition, Besonderheiten und therapeutische Entscheidungen. Der Notarzt 27(5): 223-226

Wiese CHR, Vagts DA, Kampa U, Pfeiffer G, Grom I-U, Gerth MA, Graf BM, Zausig YA, Expertengruppe Palliativ- und Notfallmedizin Akademie für Palliativmedizin und Hospizarbeit Dresden (2009b) Palliativpatienten und Patienten am Lebensende in Notfallsituationen. Anaesthesist 60: 10 

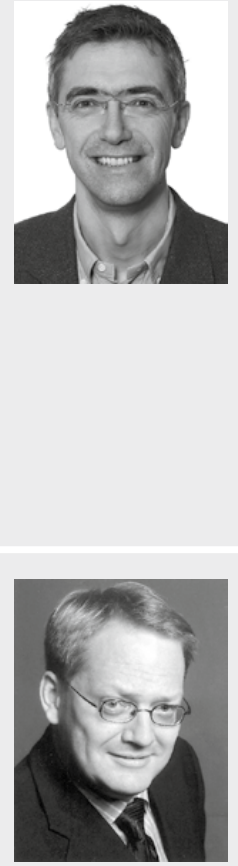

\section{Prof. Dr. iur. Stephan Rixen}

Nach Jura-Studium in Tübingen und Löwen sowie Promotion in Gießen zunächst Rechtsanwalt in Berlin, dort u.a. mit der Rechtsvertretung von Krankenhäusern aller Versorgungsstufen befasst. Ab 2001 Wissenschaftlicher Assistent am Institut für Staatsrecht und an der Forschungsstelle für das Recht des Gesundheitswesens der Universität zu Köln, dort auch Habilitation (Lehrbefugnis für Staats- und Verwaltungsrecht, deutsches und europäisches Sozialrecht sowie öffentliches Wirtschaftsrecht). Von 2007 bis 2010 Inhaber des Lehrstuhls für das Recht sozialer Dienstleistungen und Einrichtungen an der Universität Kassel. Seit 2010 Inhaber des Lehrstuhls für Öffentliches Recht, Sozialwirtschaftsund Gesundheitsrecht (Lehrstuhl für Öffentliches Recht I) an der Universität Bayreuth. Einen Forschungsschwerpunkt bildet das Gesundheitsrecht, insbesondere die Regulierung und Reform von Versorgungsstrukturen.

U.a. Mitglied des Fachbeirats (Scientific Advisory Board) des Max-Planck-Instituts für Sozialrecht und Sozialpolitik, München, des Beirats des Forschungsprojekts „Innovative Netzwerke für Dienstleistungen und Gesundheit in Regionen von morgen - INDiGeR“ (Fraunhofer Institut für Arbeitswirtschaft und Organisation, Stuttgart), des von der Deutschen Forschungsgemeinschaft (DFG) eingesetzten Gremiums „Ombudsman für die Wissenschaft“ sowie der Sachverständigenkommission für den Zweiten Gleichstellungsbericht der Bundesregierung.

\section{Prof. Dr. med. Georg Marckmann, MPH}

Studium der Medizin und Philosophie an der Universität Tübingen, PublicHealth Studium an der Harvard Universität in Boston/USA. 1992-1995 Stipendiat im DFG-Graduiertenkolleg „Ethik in den Wissenschaften“ in Tübingen. 1997 Promotion zum Dr. med. mit einer neurowissenschaftlichen Arbeit. Von 19982010 zunächst wissenschaftlicher Assistent, nach der Habilitation für das Fach „Ethik in der Medizin“ im Jahr 2003 Oberassistent und stellvertretender Direktor des Instituts für Ethik und Geschichte der Medizin der Universität Tübingen. Seit 2010 W3-Professor für Ethik, Geschichte und Theorie der Medizin an der Ludwig-Maximilians-Universität München und Leiter des gleichnamigen Instituts. Seit 2012 Präsident der Akademie für Ethik in der Medizin. 